\title{
Toxoplasma gondii infection in the context of the risk of schizophrenia development
}

\author{
EWA DUDZIŃSKA, PIOTR LISTOS*, MAGDALENA GRYZIŃSKA**, \\ HENRYK KRUKOWSKI***, BEATA TRAWIŃSKA***
}

\begin{abstract}
Chair of Public Health, Faculty of Health Sciences, Medical University of Lublin, W. Chodźki 1, 20-093 Lublin, Poland
*Department of Pathological Anatomy, Faculty of Veterinary Medicine, University of Life Sciences in Lublin, Głęboka 30, 20-033 Lublin, Poland

**Department of Biological Basis of Animal Production, ***Department of Animal Hygiene and Environment, Faculty of Biology and Animal Breeding, University of Life Sciences in Lublin, Akademicka 13, 20-950 Lublin, Poland
\end{abstract}

Dudzińska E., Listos P., Gryzińska M., Krukowski H., Trawińska B. Toxoplasma gondii infection in the context of the risk of schizophrenia development

\section{Summary}

Toxoplasma gondii ( $T$. gondii) is a protozoan parasitizing all warm-blooded mammals, including humans. The main source of infection is contact with the feces of infected animals, particularly housecats - the definitive hosts, in which $\mathrm{T}$. gondii completes its life cycle. Alternative sources of infection are contact with and consumption of contaminated meat (particularly pork), transmission from mother to fetus, and infection by oocytes present in the soil or in polluted water. T. gondii is geographically ubiquitous; its level of seroprevalence is estimated to range from about $3 \%$ in South Korea to $76 \%$ in Costa Rica.

Despite great efforts and considerable progress, toxoplasmosis remains a serious health threat worldwide. There is currently no available vaccine, and anti-toxoplasmosis drugs have substantial side effects and are not very effective. Furthermore, studies show that the parasite is able to develop resistance to them.

Many recent studies have focused on how $T$. gondii is linked to psychiatric diseases and neurocognitive processes. Research has shown a correlation between $T$. gondii infection and schizophrenia.

Keywords: protozoan, schizophrenia, toxoplasmosis

There are three infectious stages in the life cycle of T. gondii: tachyzoites, which facilitate the spread of the infection during acute infections, bradyzoites, which maintain chronic infection, and sporozoites, which are spread through the host's environment in the form of oocysts. Although infection induces a strong innate and acquired immune response controlling multiplication of the parasite, the infection is not eliminated. The host retains a lifelong latent infection characterized by the presence of cysts in the tissues. Tissue cysts become established in various cells, particularly in long-lived differentiated cells, such as neurons and muscle cells, thereby ensuring long-term infection. In humans, T. gondii has tropism for the brain, which may influence the occurrence of certain mental disorders, including schizophrenia, personality disorders, dementia, and suicidal tendencies (25).

Latent infection with $T$. gondii involves the presence of the parasite without clinical symptoms in immunocompetent patients. However, recent studies have shown a continual inflammatory state in the central nervous system following chronic $T$. gondii infection in rodents. Subtle neuromodulatory properties of the parasite in humans and mice have been the subject of recent research. Significant changes in the brain following $T$. gondii infection in animal models include activation of the glia and recruitment of peripheral immune cells to the central nervous system (14).

For this reason, studies have focused on how T. gondii is linked to psychiatric diseases and neurocognitive processes. Research has shown a correlation between $T$. gondii infection and schizophrenia. Interestingly, hallucinations, which are a key characteristic of schizophrenia, have occurred in some acute cases of $T$. gondii infection. Moreover, data from as early as 1950 showed that most hospitalized psychiatric patients tested positive for the presence of T. gondii. The culmination of these findings was a recent metaanalysis of 38 studies suggesting that $T$. gondii infection increases the risk of schizophrenia 2.7 times (19). 


\section{The role of genetic factors and infectious agents in the occurrence of symptoms of schizophrenia}

Schizophrenia is a severe mental disorder having an adverse effect on the life of patients, their families, and society. It is a neuropsychiatric disease diagnosed on the basis of positive symptoms, such as delusions and hallucinations, and negative symptoms, which are associated with a significant deterioration of the quality of life. The incidence of schizophrenia is estimated at $1 \%$ of the adult population. The onset of the disease is usually observed in late adolescence or early adulthood (11).

Studies show that genetic factors play a role in the development of schizophrenia, which is confirmed by the fact that the disease occurs in families. The latest genetic research has identified variants of genes having only a slight correlation with increased risk of the disease. Copy number variants in certain genes are associated with a greater risk, but these are very rare (5). Therefore, it has been suggested that schizophrenia may be related to non-genetic factors, such as infection by Toxoplasma gondii, which may increase the risk of the illness. According to one hypothesis, genetic susceptibility and the effect of environmental stressors increase the risk of schizophrenia, which suggests that non-genetic predisposing factors may enter into interactions with genes $(5,22)$.

Avramopoulos et al. (1) point out that many infectious agents are associated with the risk of schizophrenia, including Toxoplasma gondii (Toxo), herpes simplex virus 1 (HSV1), cytomegalovirus (CMV), and human herpes virus 6 (HHV 6). The diverse list of infectious agents suggests that these relationships may result from a reaction to the infection and the activation of the immune system, rather than from specific infectious agents. The involvement of infection and immune activation in the pathogenesis of schizophrenia indicates that genetic variants influencing the susceptibility or immune response to certain infections may determine whether or not an individual exposed to the infectious agent has an increased risk of illness (1).

\section{Relationship between $T$. gondii and modulation of neurotransmitters in the brain}

In vitro observations have shown that some antipsychotic drugs commonly used to treat schizophrenia may inhibit replication of $T$. gondii. There are several possible mechanisms by which $T$. gondii may cause symptoms of schizophrenia. First, T. gondii is capable of producing dopamine, and elevated levels of dopamine and its metabolites have been observed both in vitro and in vivo. Secondly, T. gondii may affect the production of GABA, glutamate, and serotonin, with effects depending on the strain of the parasite. Thirdly, the host immune response to T. gondii may affect expression of cytokines and the kynurenic acid pathway, both of which are implicated in the patho- genesis of schizophrenia. Finally, $T$. gondii does not induce symptoms directly, but through interactions with other factors, such as genetic susceptibility and/ or environmental factors (25).

Omar et al. (17) point out that histopathological changes in the brain in schizophrenia patients constitute evidence of infection as a cause of the disease. Their study documented that astrocytes and neurons in the brain may be infected by Toxoplasma gondii (Tg). The infection then stimulates microglia, astrocytes, and neurons to produce numerous cytokines, which in turn initiate inflammatory reactions. The parasites form cysts in the brain and produce the enzyme tyrosine hydroxylase, which is essential for dopamine production. The role of dopamine in treating schizophrenia is well documented. Tyrosine hydroxylase converts L-DOPA to dopamine. A surplus of dopamine in the pathological basis of schizophrenia is confirmed by the fact that antipsychotic drugs reduce the amount of dopamine in the brain, thereby mitigating the symptoms of schizophrenia.

Khademvatan et al. (11) show that in infected individuals the parasite influences the synthesis of neurotransmitters, particularly dopamine, which can lead to personality changes, psychotic symptoms, and in some cases neurological and psychiatric disorders. Behavioral changes and cognitive disorders have been observed in infected rodents.

In a study on rats by Fond et al. (8), the most $T$. gondii cysts in the brain were observed in the amygdalae. The infection did not affect locomotion, anxiety, hippocampus-dependent learning, fear conditioning (or its absence), or neophobia in rats. A natural predator of rats is the cat, which is also a carrier of T. gondii. Under normal conditions, rats have an aversion to cat urine, but the parasite suppresses this aversion, thereby influencing the infection cycle. Tachyzoites can penetrate different types of nerve cells, such as neurons, astrocytes, and microglial cells in the brain and Purkinje cells in the cerebellum. Intracellular tachyzoites manipulate several factors for mechanisms involved in transduction of apoptosis, antibacterial effector functions, and maturation of immune cells. Dopamine levels are $14 \%$ higher in mice with chronic infections. These neurochemical changes may be factors contributing to mental and motor disorders that accompany or are a consequence of toxoplasmosis in rodents and possibly in humans. The antipsychotic agent haloperidol and the mood stabilizer valproic acid most effectively inhibit the growth of Toxoplasma gondii in vitro with synergistic activity (8).

\section{$T$. gondif and the immune response}

Tomasik et al. (21) used a mouse model of chronic T. gondii infection and identified protein biomarkers that undergo modification in serum and brain samples at two points in time during chronic infection. In addi- 
tion, the biomarkers identified were compared with differing postmortem brain samples from 35 schizophrenia patients and 33 healthy individuals. The data obtained show that $T$. gondii caused significant immune activation, as indicated by nerve tissue damage in the animal model, which partially overlapped with the changes observed in the brains of the schizophrenia patients. The overlapping changes included increases in C-reactive protein (CRP), interleukin 1 beta (IL-1 $\beta$ ), interferon gamma (IFN- $\gamma$ ), plasminogen activator inhibitor-1 (PAI-1), tissue inhibitor of metalloproteinase-1 (TIMP-1), and vascular cell adhesion protein 1 (VCAM-1).

According to Halonen et al. (9), the immune response to $T$. gondii infection takes place through the cellular immune response, mainly by Th-1 cells, which depends on production of interleukin-12 (IL-12) and interferon gamma (IFN- $\gamma$ ). Synthesis of IL-12 is stimulated by dendritic cells (DC), neutrophils, and macrophages that are infected by the parasite at the onset of the infection and induce synthesis of IFN- $\gamma$ by $\mathrm{T}$ and NK lymphocytes. IFN- $\gamma$ is the main cytokine controlling both the acute and chronic stages of infection. IFN- $\gamma$ activates an anti-parasitic effector mechanism in both hematopoietic cells, such as macrophages, and non-hematopoietic cells, such as endothelial cells, fibroblasts, and astrocytes; both cell populations are regarded as necessary for fighting parasites. In immune-deficient hosts, such as AIDS patients, where there is a decrease in $\mathrm{CD} 4^{+} \mathrm{T}$ cells and a corresponding decrease in the level of IFN- $\gamma$, chronic infection is reactivated in the brain. In most healthy (immunocompetent) hosts, the immune response controls the replication of acute infection, and, depending on the immune response, tachyzoites differentiate into bradyzoites to form cysts, mainly in the brain and muscles. This condition is maintained throughout the lifetime of the host.

A study by Celik et al. (4) investigated the relationship between different clinical aspects of schizophrenia and a positive test for Toxoplasma gondii. The subjects of the study were 94 schizophrenia patients in whom the presence of anti-T. gondii IgG antibodies was tested by an ELISA assay. The clinical parameters studied in the patients were the type of disease, clinical condition, and awareness of the disease, which were compared with their serological status. Anti-T. gondii IgG antibodies were detected in $43(46 \%)$ patients with schizophrenia. A positive result was observed in 34 patients $(72 \%)$ with chronic schizophrenia and in 9 of those with partial remission $(22 \%)$. The incidence of latent toxoplasmosis was significantly higher in patients who were unaware of their disease (36 patients, $72 \%$ ) than in those who knew of their illness (7 patients, 16\%). The results indicate that latent toxoplasmosis may have a negative effect on the course and treatment of schizophrenia and on patients' awareness of the disease (4).

\section{The kynurenine pathway and $\boldsymbol{T}$. gondii}

Toxoplasma gondii, a protozoan and internal parasite, has a strong predilection for infecting the central nervous system and is associated with increased prevalence of numerous psychiatric diseases (18). Several in vitro and in vivo studies have shown that infection with $T$. gondii can affect the structure, bioenergetics, and function of brain cells and modifies numerous cellular processes in the host, including dopaminergic, tryptophan-kynurenine, GABAergic, AKT1, Jak/STAT, and vasopressinergic pathways. These mechanisms, underlying neuropathology in latent toxoplasmosis, also seem to be active in schizophrenia, and therefore it is important to investigate connections between these two disorders (6).

In a study on mice, Notarangelo et al. (15) found that $T$. gondii infection leads to degradation of tryptophan along the kynurenine pathway (KP), which contains several neuroactive metabolites, including 3-hydroxykinurenine (3-HK), quinolinic acid (QUINN), and kynurenic acid (KYNA). Because these endogenous compounds may be a link between T. gondii and the occurrence of schizophrenia symptoms, KP metabolites were measured in mice 8 and 28 days after infection with $T$. gondii. The infected mice initially exhibited a reduced level of tryptophan in the brain and blood serum, but not in the liver. These results were associated with elevated levels of kynurenine, KYNA, 3-HK, and quinolinic acid in the brain. The most significant increases in these KP metabolites were observed in the brain 28 days after infection. It is worth noting that the antiparasitic drugs pyrimethamine and sulfadiazine, the standard treatment for toxoplasmosis, substantially lower 3-HK and KYNA levels in the brains of infected mice when applied from 28 to 56 days after infection. The results of the study indicate that $T$. gondii infection, probably through the activation of the microglia and astrocytes, increases the production of KP metabolites in the brain, which may be linked to the occurrence of symptoms typical of schizophrenia (15).

A recent study by Okusaga et al. (16) shows that chronic Toxoplasma gondii infection and an elevated level of kynurenine (KYN) are correlated with the incidence of suicidal behavior in schizophrenia patients. The researchers studied 950 schizophrenia patients to investigate the possibility of a link between a positive $T$. gondii result, a history of suicide attempts, and the plasma level of kynurenine in patients with schizophrenia. The results suggest that $T$. gondii and KYN may affect the risk of suicidal behavior in individuals with schizophrenia.

Patients infected with $T$. gondii have a chronic infection that lasts for their entire lives. The infection is usually asymptomatic and is characterized by the formation of inactive cysts with bradyzoites located in the tissues. However, in individuals with reduced 
immunity or congenital infection, toxoplasmosis may develop into a very severe form (24).

An increasing number of studies indicate that the neurotrophic parasite Toxoplasma gondii is involved in the development of psychiatric disorders $(2,13,20)$.

In the last 10 years many independent studies have shown that this parasitic disease may be indirectly responsible for hundreds of thousands of deaths due to its effect on the incidence of traffic and workplace accidents and of suicides. Furthermore, latent toxoplasmosis may be one of the most important risk factors for schizophrenia. These effects may be due to active manipulation of important dopamine neurotransmitters or tryptophan metabolites by the parasite Toxoplasma gondii (7).

Analysis of the studies described above suggests that T. gondii is a likely cause of certain aspects of mental disorders, including schizophrenia. Hence it is likely that schizophrenia may also be caused by pathogens and vectors originating in domestic animals. This knowledge may prove extremely useful in discovering the pathogenesis of this enigmatic illness. It may also contribute to a framework for the development of new methods for treating and preventing the disease (10). Although the removal of the pathogen cannot be expected to eliminate or inhibit the development of schizophrenia, prevention of toxoplasmosis may affect the prevalence of the disease. It may also be concluded that if toxoplasmosis were to be included on the list of diseases for which the law requires registration of all cases in cats and other domestic animals, the prevalence of this disease might decline in humans (3). tial.

Further research to confirm this hypothesis is essen-

\section{References}

1. Avramopoulos D., Pearce B. D., McGrath J., Wolyniec P., Wang R., Eckart N., Hatzimanolis A., Goes F. S., Nestadt G., Mulle J., Coneely K., Hopkins M., Ruczinski I., Yolken R., Pulver A. E.: Infection and inflammation in schizophrenia and bipolar disorder: a genome wide study for interactions with genetic variation. PLoS One 2015, 10, e0116696.

2. Berenreiterová M., Flegr J., Kuběna A. A., Němec P.: The Distribution of Toxoplasma gondii Cysts in the Brain of a Mouse with Latent Toxoplasmosis: Implications for the Behavioral Manipulation Hypothesis. PLoS One 2011, 6 , e28925.

3. Carter C. J.: Toxoplasmosis and Polygenic Disease Susceptibility Genes: Extensive Toxoplasma gondii Host/Pathogen Interactome Enrichment in Nine Psychiatric or Neurological Disorders. J. Pathog. 2013, 965046.

4. Celik T., Kartalci S., Aytas O., Akarsu G. A., Gozukara H., Unal S.: Association between latent toxoplasmosis and clinical course of schizophrenia-continuous course of the disease is characteristic for Toxoplasma gondii-infected patients. Folia Parasitol. (Praha) 2015, 1, 62

5. Eells J. B., Varela-Stokes A., Guo-Ross S. X., Kummari E., Smith H. M., Cox E., Lindsay D. S.: Chronic Toxoplasma gondii in Nurr1-null heterozygous mice exacerbates elevated open field activity. PLoS One 2015, 10, e0119280.

6. Elsheikha H. M., Büsselberg D., Zhu X. Q.: The known and missing links between Toxoplasma gondii and schizophrenia. Metab. Brain. Dis. 2016.

7. Flegr J.: How and why Toxoplasma makes us crazy. Trends Parasitol. 2013, 29, 156-163.

8. Fond G., Capdevielle D., Macgregor A., Attal J., Larue A., Brittner M., Ducasse D., Boulenger J. P.: Toxoplasma gondii: a potential role in the genesis of psychiatric disorders. Encephale. 2013, 39, 38-43.

9. Halonen S. K., Weiss L. M.: Toxoplasmosis. Handb. Clin. Neurol. 2013, 114, 125-145.
10. He J.J., Ma J., Elsheikha H. M., Song H. Q., Zhou D. H., Zhu X. Q.: Proteomic Profiling of Mouse Liver following Acute Toxoplasma gondii Infection. PLoS One 2016, 11, e0152022.

11. Khademvatan S., Khajeddin N., Izadi S., Yousefi E.: Investigation of anti-Toxocara and anti-toxoplasma antibodies in patients with schizophrenia disorder. Schizophr. Res. Treatment. 2014, 230349.

12. Khademvatan S., Saki J., Khajeddin N., Izadi-Mazidi M. Beladi R., Shafiee B., Salehi Z.: Toxoplasma gondii Exposure and the Risk of Schizophrenia. Jundishapur J. Microbiol. 2014, 7, e12776.

13. Markovitz A. A., Simanek A. M., Yolken R. H., Galea S., Koenen K. C., Chen S., Aiello A. E.: Toxoplasma gondii and anxiety disorders in a community-based sample. Brain Behav. Immun. 2015, 43, 192-197.

14. Möhle L., Israel N., Paarmann K., Krohn M., Pietkiewicz S., Müller A., Lavrik I. N., Buguliskis J. S., Schott B. H., Schlüter D., Gundelfinger E.D., Montag D., Seifert U., Pahnke J., Dunay I. R.: Chronic Toxoplasma gondii infection enhances $\beta$-amyloid phagocytosis and clearance by recruited monocytes. Acta Neuropathol. Commun. 2016, 4, 25.

15. Notarangelo F. M., Wilson E. H., Horning K. J., Thomas M. A., Harris T. H., Fang $Q$., Hunter C. A., Schwarcz R.: Evaluation of kynurenine pathway metabolism in Toxoplasma gondii-infected mice: implications for schizophrenia. Schizophr. Res. 2014, 152, 261-267.

16. Okusaga O., Duncan E., Langenberg P., Brundin L., Fuchs D., Groer M. W., Giegling I., Stearns-Yoder K. A., Hartmann A. M., Konte B., Friedl M., Brenner L. A., Lowry C. A., Rujescu D., Postolache T. T.: Combined Toxoplasma gondii seropositivity and high blood kynurenine--Linked with nonfatal suicidal selfdirected violence in patients with schizophrenia. J. Psychiatr. Res. 2016, 72, 74-81.

17. Omar A., Bakar O. C., Adam N. F., Osman H., Osman A., Suleiman A. H., Manaf M. R., Selamat M. I.: Seropositivity and serointensity of Toxoplasma gondii antibodies and DNA among patients with schizophrenia. Korean J. Parasitol. 2015, 53, 29-34

18. Passeri E., Jones-Brando L., Bordón C., Sengupta S., Wilson A. M., Primerano A. Rapoport J. L., Ishizuka K., Kano S., Yolken R. H., Sawa A.: Infection and characterization of Toxoplasma gondii in human induced neurons from patients with brain disorders and healthy controls. Microbes. Infect. 2016, 18, 153-158.

19. Sugden K., Moffitt T. E., Pinto L., Poulton R., Williams B. S., Caspi A.: Is Toxoplasma Gondii Infection Related to Brain and Behavior Impairments in Humans? Evidence from a Population-Representative Birth Cohort. PLoS One 2016, 11, e0148435.

20. Sutterland A. L., Fond G., Kuin A., Koeter M. W., Lutter R., van Gool T., Yolken R., Szoke A., Leboyer M., de Haan L.: Beyond the association. Toxoplasma gondii in schizophrenia, bipolar disorder, and addiction: systematic review and meta-analysis. Acta Psychiatr. Scand. 2015, 132, 161-179.

21. Tomasik J., Schultz T. L., Kluge W., Yolken R. H., Bahn S., Carruthers V. B.: Shared Immune and Repair Markers During Experimental Toxoplasma Chronic Brain Infection and Schizophrenia. Schizophr. Bull. 2016, 42, 386-395.

22. Torrey E. F., Bartko J. J., Yolken R. H.: Toxoplasma gondii and other risk factors for schizophrenia: an update. Schizophr. Bull. 2012, 38, 642-647.

23. Webster J. P., Kaushik M., Bristow G. C., McConkey G. A.: Toxoplasma gondii infection, from predation to schizophrenia: can animal behaviour help us understand human behaviour? J. Exp. Biol. 2013, 216, 99-112.

24. Witola W. H., Liu S. R., Montpetit A., Welti R., Hypolite M., Roth M., Zhou Y., Mui E., Cesbron-Delauw M. F., Fournie G. J., Cavailles P., Bisanz C., Boyer K., Withers S., Noble A. G., Swisher C. N., Heydemann P. T., Rabiah P., Muench S. P., McLeod R.: ALOX12 in human toxoplasmosis. Infect. Immun. 2014, $82,2670-2679$

25. Xiao J., Yolken R. H.: Strain hypothesis of Toxoplasma gondii infection on the outcome of human diseases. Acta Physiol. (Oxf). 2015, (4), 828-845

Corresponding author: Ewa Dudzińska, PhD, Chodźki 1, 20-093 Lublin, Poland; tel. +48814486720 